\title{
Left atrial expansion index is associated with recurrent stroke
}

\author{
Shih-Hung Hsiao', 2 (1) \\ ${ }^{1}$ Division of Cardiology, Department of Internal Medicine, E-Da Hospital, I-Shou University; Kaohsiung-Taiwan \\ ${ }^{2}$ School of Medicine, National Yang-Ming University; Taipei-Taiwan
}

\section{ABSTRACT}

Objective: Although left atrial (LA) expansion index is associated with cardiovascular prognosis, whether it affects recurrent strokes is still unknown.

Methods: This study enrolled 176 patients hospitalized with first ischemic stroke. Their stroke subtypes were classified as cardioembolic stroke (CE), noncardioembolic stroke (NCE), embolic stroke of undetermined source (ESUS), or transient ischemic attack. The LA expansion index was calculated as $\left(\mathrm{Vol}_{\max }-\mathrm{Vol}_{\min }\right) \times 100 \% / \mathrm{Vol}_{\min }$, where $\mathrm{Vol}_{\max }$ was defined as maximal LA volume and $\mathrm{Vol}_{\min }$ as minimal LA volume. The study endpoint was recurrent ischemic stroke.

Results: Over a five-year (mean 4.9 years) follow-up period, 21 (11.9\%) participants reached the study endpoint, including 10 with CE, five with NCE, and six with ESUS. The LA expansion index was lower in the event groups compared with the non-event group. For predicting recurrent stroke, LA expansion index $<62.5 \%$ ( $76 \%$ sensitivity and $68 \%$ specificity) was superior to LA volume and E/e'. Kaplan-Meier curves revealed that the five-year cumulative recurrent stroke rate in patients with LA expansion index $<62.5 \%$ was $23.9 \%$, which was significantly higher than the five-year cumulative recurrent stroke rate of $4.6 \%$ in patients with LA expansion index $>62.5 \%$ (log rank $p<0.001)$. The LA expansion index was a significant independent predictor of recurrent stroke (hazard ratio $=0.873 ; 95 \%$ confidence interval: $0.790-0.973$ per $10 \%$ increase in LA expansion index; $\mathrm{p}=0.009$ ).

Conclusion: The LA expansion index is useful for predicting recurrent stroke.

Keywords: Left atrial expansion index, recurrent stroke, atrial fibrillation

Cite this article as: Hsiao SH. Left atrial expansion index is associated with recurrent stroke. Anatol J Cardiol 2021; 25: 484-90.

\section{Introduction}

Stroke is characterized by high morbidity and a high recurrence rate, therefore posing a significant burden on healthcare. Population-based studies reveal that left atrial (LA) dilation is a marker of cardiovascular disease and ischemic stroke (1-4). Left atrial dilation with atrial fibrosis is also associated with the presence and persistence of atrial fibrillation (AF) $(5,6)$. Left atrial volume is a predictor of stroke regardless of $A F$, and a high $L A$ volume is linked to cardioembolic stroke (CE) and embolic stroke with undetermined source (ESUS) (7). For predicting recurrent stroke, moderate-to-severe LA enlargement was also an independent marker of recurrent CE and ESUS in a multiethnic cohort (8). As an outcome predictor, LA function outperforms LA diameter and LA volume $(9,10)$. The LA expansion index has a logarithmic correlation with left ventricular (LV) filling pressure and is a useful outcome predictor in patients with cardiovascular disease (11). In addition, the occurrence of $A F$ in the general population and in high-risk patients post bypass is associated with low LA expansion index $(12,13)$. In this study, we tested our hypothesis that similar to LA volume, the LA expansion index in patients with stroke can be used to identify those at high risk for recurrent stroke after a first stroke event. 


\section{HIGHLIGHTS}

- Left atrial (LA) expansion index is useful for identifying patients at high risk for recurrent stroke after the first stroke event.

- LA expansion index $<62.5 \%$ is superior to LA volume and $\mathrm{E} / \mathrm{e}^{\prime}$ for predicting recurrent stroke.

- Five-year recurrent stroke rate in patients with LA expansion index $<62.5 \%$ is $23.9 \%$, which is significantly higher than $4.6 \%$ in patients with LA expansion index $>62.5 \%$.

\section{Methods}

\section{Study population}

This prospective study recruited 176 patients with a history of a first embolic stroke event. Stroke subtype was prospectively assigned by the attending neurologist on the basis of consensus criteria for embolic stroke with undetermined source (14). Accordingly, ischemic stroke was divided into three subtypes: CE, noncardioembolic stroke (NCE), and ESUS. For ischemic strokes with multiple possible causes, the subtype was determined according to the consensus of the attending neurologists. Transient ischemic attack (TIA) was diagnosed if these clinical signs recovered rapidly with no indicatioin of brain infarction on neuro-imaging. The 176 patients with stroke included 51 with CE, 36 with NCE, 53 with ESUS, and 36 with TIA. At enrollment, creatinine clearance $(\mathrm{CCr})$ was estimated by the Cockroft-Gault equation, and renal dysfunction was defined as $\mathrm{CCr}<60 \mathrm{~mL} /\left(\min \times 1.73 \mathrm{~m}^{2}\right)(15)$. The endpoint of this study was recurrent ischemic stroke. The study protocol was approved by the Institutional Review Board of Kaohsiung Veterans General Hospital. All participants gave written informed consent before participating in the study.

\section{Echocardiographic measurements}

Pulsed-wave tissue Doppler was performed in apical view, and a pulsed-wave Doppler sample volume was placed at the level of the mitral annulus over the septal and lateral borders. The average early-diastolic velocity (e') of the septal and lateral mitral annuli was used to estimate LV filling pressure by E/e' method. An E/e' $>15$ was interpreted as increased LV filling pressure (16). All LA volume measurements were calculated by biplane area-length method in apical four- and two-chamber views (17). The LA volume was measured at two points, immediately before mitral valve opening (maximal LA volume or $\mathrm{Vol}_{\max }$ ) and at mitral valve closure (minimal LA volume or $\mathrm{Vol}_{\min }$ ). The LA expansion index was calculated as $\left(\mathrm{Vol}_{\max }-\mathrm{Vol}_{\min }\right) \times 100 \% / \mathrm{Vol}-$ min. For patients with AF, LA expansion index was calculated as the average of measurements in five consecutive beats (11). In all the patients, LA volume was indexed to the body surface area. An indexed Vol max larger than $34 \mathrm{~mL} / \mathrm{m}^{2}$ was defined as LA enlargement (18).

\section{Clinical follow-up}

Participants were followed up at the cardiovascular clinic every three months. The mean duration of follow-up was 4.9 years, and the study endpoint was occurrence of a recurrent ischemic stroke. All patients with recurrent stroke underwent standard ischemic stroke evaluations at index hospitalization, including biochemical studies, neuroimaging (computed tomography/magnetic resonance imaging, carotid ultrasound, and transcranial Doppler ultrasound), 12-lead electrocardiogram, transthoracic (or transesophageal) echocardiography, and Holter study (minimum 24 hours). Recurrent stroke subtype was prospectively assigned by the attending neurologist according to consensus criteria for ESUS (14).

\section{Detection of new atrial fibrillation and heart failure}

A resting ECG was obtained at every visit throughout the follow-up period. Participants were encouraged to visit the outpatient clinic for any feeling of palpitation or irregular heartbeat. At every unscheduled visit for palpitation or irregular heartbeat, resting and 24-hour Holter ECGs were performed to detect AF. Medical assistants reviewed medical records once every three months. Newly diagnosed AF and heart failure (HF) were recorded. Diagnoses of AF were confirmed by the participating cardiologists (Hsiao SH). An HF was defined as a hospital stay of at least one night for treatment of a clinical syndrome with at least two of the following symptoms: paroxysmal nocturnal dyspnea, orthopnea, elevated jugular venous pressure, pulmonary rales, a third heart sound, pulmonary edema on chest radiography, or low cardiac output with inadequate peripheral perfusion.

\section{Interobserver variability}

In the first 50 patients who enrolled, images were acquired by two independent sonographers using the same imaging protocol. Each acquisition was analyzed by two different observers. The LA expansion index was also measured by two independent observers. Interobserver variability was calculated as the absolute difference between the values obtained by the two observers divided by the mean. Interobserver variability of LA expansion index was $4.1 \pm 4.9 \%$.

\section{Statistical analysis}

The Statistical Package for Social Sciences software was used for all statistical analyses. Baseline characteristics and echocardiographic parameters were analyzed according to the presence or absence of recurrent ischemic stroke. All continuous variables were presented as means \pm standard deviation (SD). The Shapiro-Wilk test was applied to test normal distribution of study population. Data reported as mean \pm SD were compared with the independent samples t test, clinical characteristics were compared using the Mann-Whitney $U$ test for continuous variables, and clinical characteristics were compared using the chi-squared analysis of categorical variables. A $p$ value of $<0.05$ was considered statistically significant. Analyses of five-year cumulative recurrent ischemic strokes were performed according to the quartile of LA expansion index. Nested 
repeated analyses of variance were applied to assess the difference of LA expansion index between recurrent and no recurrent stroke according to the presence of $\mathrm{AF}$ or not. The area under receiver operating characteristic (ROC) curve (AUC) was used to evaluate the sensitivity and specificity of recurrent ischemic stroke predictors. The $\mathrm{C}$-statistic was calculated to compare $\mathrm{E} / \mathrm{e}^{\prime}$, maximal indexed LA volume, and LA expansion index in terms of accuracy in predicting recurrent strokes. Kaplan-Meier curves depicted the cumulative recurrent stroke rate according to LA expansion index. Cox proportional hazards regression models were used to analyze outcomes according to time-toevent data and to analyze associations between recurrent ischemic strokes and LA expansion index while controlling for baseline characteristics and echocardiographic parameters showing signific ant $(p<0.05)$ associations with events. Otherwise, Cox proportional hazards regression models were also used to analyze the prognostic impact of LA expansion index after excluding patients with AF.

\section{Results}

Table 1 lists the basic characteristics of the participants according to recurrent ischemic stroke events. Over a mean follow-up time of 4.9 years, $21(11.9 \%)$ participants reached the study endpoint of recurrent ischemic stroke. In the 21 patients with recurrent ischemic stroke, $10(47.6 \%)$ had $\mathrm{CE}$, five $(23.8 \%)$ had NCE, and six (28.6\%) had ESUS. Male sex, diabetes, AF, and $\mathrm{HF}$ were more common in the recurrent stroke group than in the non-recurrent stroke group. Regarding echocardiographic parameters, the recurrent stroke group had larger minimal indexed LA volumes and lower LA expansion indices than the non- recurrent stroke group. The recurrent stroke group had more frequent $A F$, which resulted in a higher rate of prescriptions for non-vitamin $\mathrm{K}$ antagonist oral anticoagulant. Table 2 shows $L A$ expansion index according to recurrent stroke and AF. Patients with recurrent stroke and AF had the lowest of LA expansion index. During the follow-up period, LA expansion indices went progressively downhill. Patients with recurrent stroke went more downhill than those without recurrent stroke, particularly in those with AF.

The ROC curves in Figure 1 show that LA expansion index was a significantly better predictor of recurrent stroke than maximal indexed LA volume and $\mathrm{E} / \mathrm{e}^{\prime}$ (all $\mathrm{p}<0.0001$ ). The best cutoff was LA expansion index $<62.5 \%$ (sensitivity $76 \%$, specificity $68 \%$, and AUC 0.775). The Kaplan-Meier curves in Figure 2 indicate that the five-year cumulative recurrent stroke rate was $23.9 \%$ in patients with LA expansion $<62.5 \%$, but only $4.6 \%$ in patients with LA expansion index $>62.5 \%(p<0.001)$. Figure 3 depicts the inverse relationship between recurrent stroke and the quartile of LA expansion index. In the lowest quartile of LA expansion index $(15 \%-53 \%)$, five-year cumulative recurrent stroke rate reached $27.3 \%$.

According to univariate analyses, significant predictors of recurrent stroke included age, AF, and LA expansion index. Table 3 shows that the independent predictors identified in multiple

\begin{tabular}{|c|c|c|c|}
\hline Variables & $\begin{array}{l}\text { No recurrent } \\
\text { stroke } n=155\end{array}$ & $\begin{array}{c}\text { Recurrent } \\
\text { stroke } n=21\end{array}$ & $P$-value \\
\hline Age (years) & $68 \pm 14$ & $73 \pm 9$ & 0.118 \\
\hline Male sex (\%) & $104(67.1 \%)$ & $18(85.7 \%)$ & 0.012 \\
\hline Hypertension (\%) & $123(79.4 \%)$ & $17(81.0 \%)$ & 0.783 \\
\hline Diabetes (\%) & $49(31.6 \%)$ & $10(47.6 \%)$ & 0.012 \\
\hline Current tobacco use (\%) & $65(41.9 \%)$ & $9(42.9 \%)$ & 0.766 \\
\hline Renal dysfunction (\%) & $36(23.2 \%)$ & $6(28.6 \%)$ & 0.107 \\
\hline Atrial fibrillation (\%) & $28(18.1 \%)$ & $8(38.1 \%)$ & 0.001 \\
\hline Heart failure (\%) & $20(12.9 \%)$ & $5(23.8 \%)$ & 0.013 \\
\hline Total cholesterol (mg/dL) & $186 \pm 43$ & $179 \pm 38$ & 0.535 \\
\hline LDL-cholesterol (mg/dL) & $105 \pm 34$ & $105 \pm 34$ & 0.976 \\
\hline $\mathrm{CHA}_{2} \mathrm{DS}_{2}$-VASc score & $4.9 \pm 2.9$ & $5.1 \pm 2.7$ & 0.315 \\
\hline NIHSS during stroke & $9.0 \pm 6.9$ & $9.3 \pm 8.3$ & 0.426 \\
\hline $\operatorname{LVEF}(\%)$ & $55 \pm 10$ & $51 \pm 11$ & 0.147 \\
\hline$E / e^{\prime}$ & $11.9 \pm 3.1$ & $11.8 \pm 3.1$ & 0.915 \\
\hline $\begin{array}{l}\text { Maximal indexed LAV } \\
\left(\mathrm{mL} / \mathrm{m}^{2}\right)\end{array}$ & $36 \pm 14$ & $42 \pm 20$ & 0.062 \\
\hline $\begin{array}{l}\text { Minimal indexed LAV } \\
\left(\mathrm{mL} / \mathrm{m}^{2}\right)\end{array}$ & $21 \pm 10$ & $31 \pm 19$ & 0.002 \\
\hline LA expansion index (\%) & $75 \pm 27$ & $40 \pm 20$ & $<0.0001$ \\
\hline First stroke type & & & 0.011 \\
\hline Cardioembolic & $43(27.7 \%)$ & $8(38.1 \%)$ & \\
\hline Noncardioembolic & $32(20.6 \%)$ & $4(19.0 \%)$ & \\
\hline ESUS & $47(30.3 \%)$ & $6(28.6 \%)$ & \\
\hline TIA & $33(21.3 \%)$ & $3(14.3 \%)$ & \\
\hline \multicolumn{4}{|l|}{ Recurrent stroke type } \\
\hline Cardioembolic & & $10(47.6 \%)$ & \\
\hline Noncardioembolic & & $5(23.8 \%)$ & \\
\hline ESUS & & $6(28.6 \%)$ & \\
\hline TIA & & $0(0 \%)$ & \\
\hline \multicolumn{4}{|l|}{ Medications } \\
\hline Aspirin & $126(81.3 \%)$ & $18(85.7 \%)$ & 0.523 \\
\hline Clopidogrel & $108(69.7 \%)$ & $13(61.9 \%)$ & 0.364 \\
\hline NOAC & $30(19.4 \%)$ & $9(42.9 \%)$ & $<0.0001$ \\
\hline Statin & $121(78.1 \%)$ & $17(81.0 \%)$ & 0.546 \\
\hline
\end{tabular}

E/e' - peak early-diastolic mitral inflow/ peak early-diastolic mitral annular velocity; ESUS - embolic stroke of undetermined source; LA - left atrial; LAV - left atrial volume; LDL - low-density lipoprotein; LVEF - left ventricular ejection fraction; NIHSS - National Institute of Health Stroke Scale; NOAC - non-vitamin K antagonist oral anticoagulant; TIA transient ischemic attack

Cox regression analyses were $A F$ and $L A$ expansion index. A $10 \%$ increase in LA expansion index was associated with a $13 \%$ decrease in the five-year recurrent stroke rate [Hazard ratio (HR) 0.873; $p=0.009$; $95 \%$ confidence interval (CI): 0.790-0.973]. 


\begin{tabular}{|c|c|c|c|c|c|c|c|c|}
\hline \multirow[b]{2}{*}{ Variable } & \multicolumn{2}{|c|}{ No recurrent stroke } & \multirow[b]{2}{*}{$\boldsymbol{P}$-value } & \multicolumn{2}{|c|}{ Recurrent stroke } & \multirow[b]{2}{*}{$P$-value } & \multirow{2}{*}{$\begin{array}{l}P \text {-value of no AF } \\
\text { between no recurrent } \\
\text { and recurrent stroke }\end{array}$} & \multirow{2}{*}{$\begin{array}{l}P \text {-value of AF } \\
\text { between no recurrent } \\
\text { and recurrent stroke }\end{array}$} \\
\hline & No AF & $\mathbf{A F}$ & & No AF & $\mathbf{A F}$ & & & \\
\hline $\begin{array}{l}\text { LA expansion index } \\
\text { at enrollment }\end{array}$ & $76 \pm 28 \%$ & $70 \pm 27 \%$ & 0.478 & $42 \pm 23 \%$ & $33 \pm 16 \%$ & 0.096 & $<0.001$ & $<0.001$ \\
\hline $\begin{array}{l}\text { LA expansion index } \\
\text { at end* }\end{array}$ & $75 \pm 24 \%$ & $67 \pm 24 \%$ & 0.286 & $38 \pm 18 \%$ & $25 \pm 15 \%$ & 0.011 & $<0.001$ & $<0.001$ \\
\hline
\end{tabular}

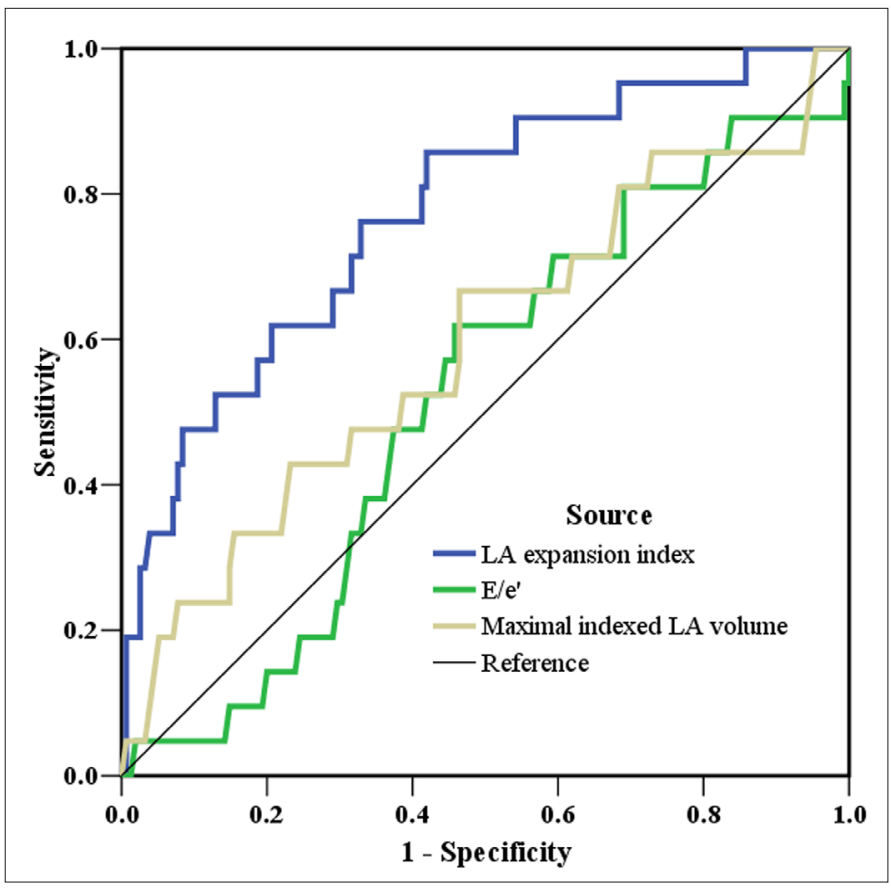

Figure 1. The receiver operating characteristic curves for left atrial expansion index, maximal indexed LA volume, and E/e' for predicting five-year cumulative recurrent stroke; LA expansion index vs. maximal indexed LA volume, $P<0.001$; LA expansion index vs. E/e', $P<0.001$; maximal indexed $L A$ volume vs. E/e', P0.402

Table 4 shows multiple Cox regression analyses after excluding $A F$, and $L A$ expansion index remains a significant predictor of recurrent stroke (HR 0.892; $\mathrm{p}=0.041 ; 95 \% \mathrm{Cl}$ : 0.801-0.988).

\section{Discussion}

The main finding of this study is that LA expansion index is associated with recurrent stroke events. An inverse relationship was observed between LA expansion index and the cumulative risk of a recurrent stroke within five years. Although maximal indexed LA volume is also useful for predicting stroke recurrence (maximal indexed LA volume $>32.5 \mathrm{~mL} / \mathrm{m}^{2}$ had sensitivity $67 \%$, specificity $54 \%$, and AUC 0.594$)$, LA expansion index was a significantly better predictor than maximal indexed LA volume (AUC 0.775 vs. $0.594, p<0.001$ ). According to Yaghi et al. (8), moderate-to-severe LA enlargement is an independent risk factor for recurrent CE and ESUS but is not a risk factor for total

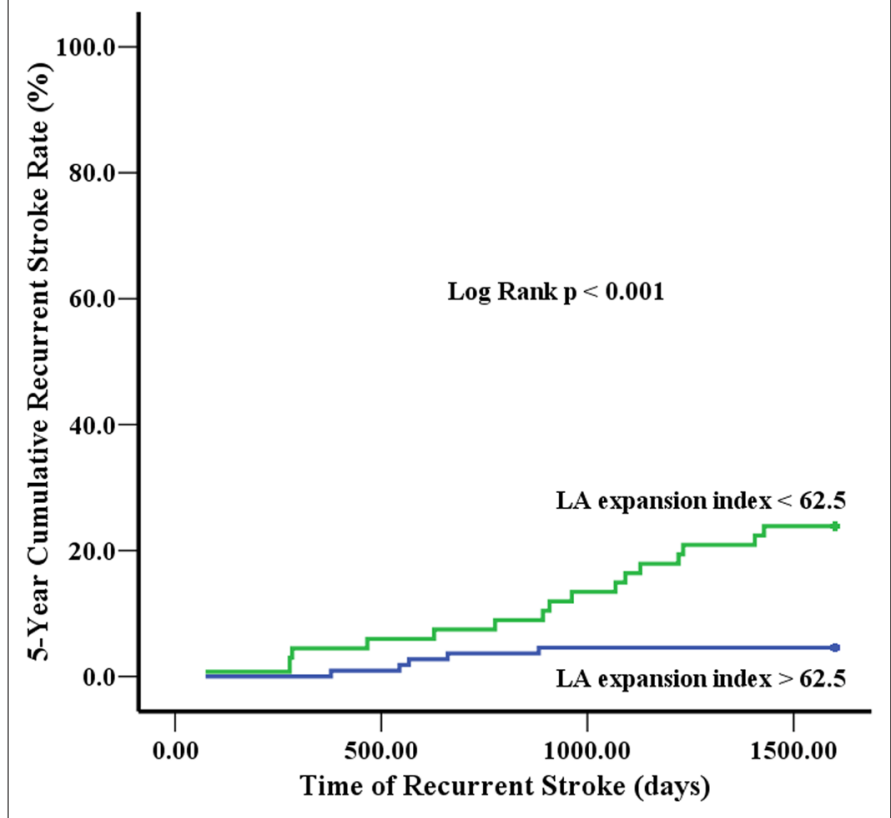

Figure 2. Kaplan-Meier curves according to LA expansion index > or $<62.5 \%$

recurrent ischemic stroke. Mild LA enlargement is not associated with recurrent ischemic stroke. A limitation of the study by Yaghi et al. (8) was that the LA diameter measurement was not standardized, and accurately determining the edge-to-edge diameter in parasternal long-axis view was problematic. Another limitation was that LA size was estimated by qualitative assessment when accurate measurements were not possible. Therefore, the result should be interpreted cautiously. In another study in China, LA diameter indexed to height or body surface area revealed that it was an independent predictor of recurrence of either CE or cryptogenic stroke and also an independent predictor of recurrence of all ischemic strokes (19). In patients with stroke and with nonvalvular $A F$, the LA diameter is also associated with recurrent stroke risk (20). Similar to previous studies, LA expansion index, which is a parameter of LA function, is better than LA volume in predicting recurrent stroke.

The association between LA function and recurrent CE is intuitive as LA dysfunction promotes thrombus formation, which causes embolic events. Left atrial fibrosis plays an integral role in the thrombogenesis of the LA substrate, which leads to recurrent $\mathrm{CE}$ regardless of AF. Thus, LA expansion 


\begin{tabular}{|c|c|c|c|c|}
\hline \multirow{2}{*}{ Variables } & \multirow{2}{*}{$\begin{array}{c}\text { Univariate analysis } \\
\text { Hazard ratio } \\
\text { (95\% confidence interval) }\end{array}$} & \multirow{2}{*}{\multicolumn{3}{|c|}{$\begin{array}{l}\text { Multiple Cox regression analysis } \\
\text { Hazard ratio }\end{array}$}} \\
\hline & & & & \\
\hline Age (years) & $\begin{array}{c}1.028(1.001-1.056) \text { per } 1 \text { year } \\
\text { increase }\end{array}$ & 0.046 & $\begin{array}{l}0.999(0.963-1.036) \text { per } 1 \text { year } \\
\text { increase }\end{array}$ & 0.950 \\
\hline Diabetes & $1.763(0.946-3.288)$ & 0.096 & $2.003(0.792-5.069)$ & 0.142 \\
\hline Hypertension & $1.033(0.554-1.925)$ & 0.819 & $1.198(0.416-3.449)$ & 0.738 \\
\hline Atrial fibrillation & $2.256(1.318-5.034)$ & 0.006 & $2.360(0.725-7.684)$ & 0.154 \\
\hline Heart failure & $1.903(0.935-3.872)$ & 0.086 & $1.578(0.964-2.978)$ & 0.142 \\
\hline Left atrial expansion index (\%) & $0.842(0.754-0.940)$ per $10 \%$ increase & 0.002 & $0.873(0.790-0.973)$ per $10 \%$ increase & 0.009 \\
\hline
\end{tabular}

\begin{tabular}{|c|c|c|c|c|}
\hline \multirow{2}{*}{ Variables } & \multirow{2}{*}{$\begin{array}{c}\text { Univariate analysis } \\
\text { Hazard ratio } \\
(95 \% \text { confidence interval) }\end{array}$} & \multirow{2}{*}{\multicolumn{3}{|c|}{$\begin{array}{l}\text { Multiple Cox regression analysis } \\
\text { Hazard ratio }\end{array}$}} \\
\hline & & & & \\
\hline Age (years) & $\begin{array}{l}1.021(1.001-1.042) \\
\text { per } 1 \text { year increase }\end{array}$ & 0.049 & $\begin{array}{l}1.010(0.989-1.041) \\
\text { per } 1 \text { year increase }\end{array}$ & 0.485 \\
\hline Hypertension & $1.295(0.450-3.727)$ & 0.632 & $0.968(0.303-30.90)$ & 0.956 \\
\hline Heart failure & $1.287(0.370-5.224)$ & 0.820 & $1.074(0.295-4.327)$ & 0.765 \\
\hline Left atrial expansion index (\%) & $\begin{array}{c}0.856(0.776-0.986) \\
\text { per } 10 \% \text { increase }\end{array}$ & 0.026 & $\begin{array}{l}0.892(0.801-0.988) \\
\text { per } 10 \% \text { increase }\end{array}$ & 0.041 \\
\hline
\end{tabular}

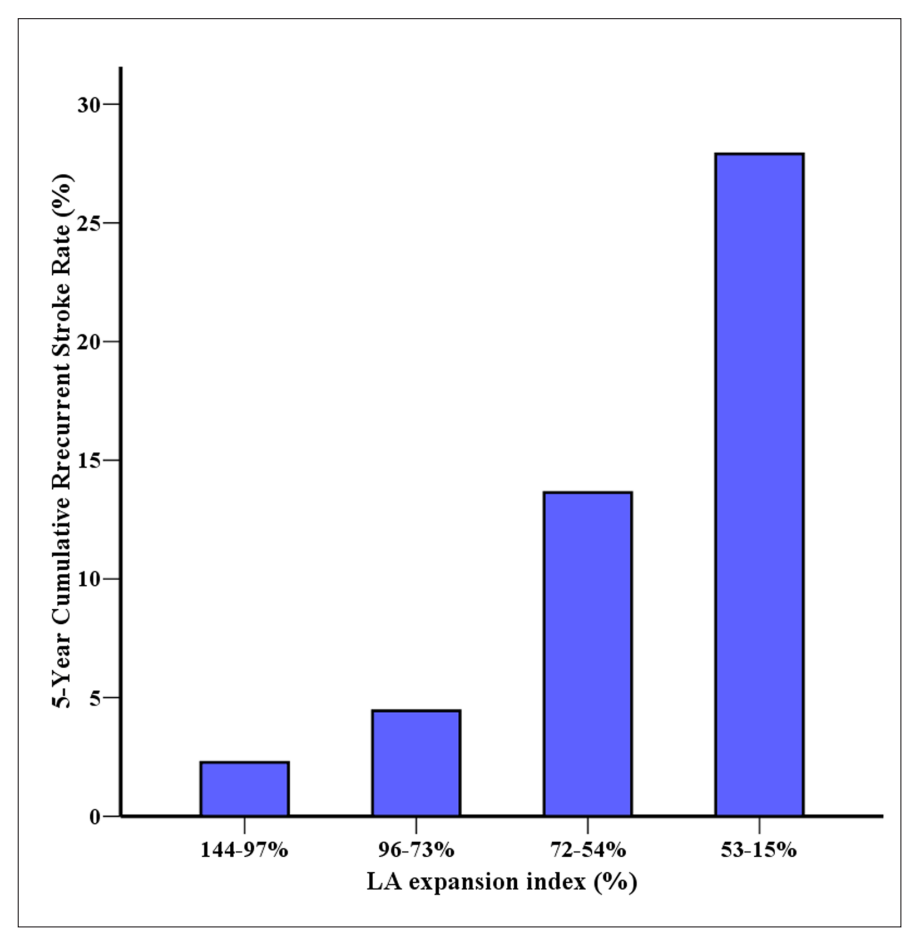

Figure 3. The five-year cumulative rate of recurrent stroke by quartile of left atrial expansion index index became a significant predictor of recurrent CE. Studies of thrombi collected during intracranial mechanical recanalization in patients with stroke and with large-vessel occlusion indicate that ESUS is usually cardioembolic (21). Recent reports that ESUS correlates with atrial fibrosis in patients without AF also help to explain why the LA expansion index has a high predictive power for recurrent ESUS (22). In terms of NCE, its causes such as intracranial, extracranial or aortic atheroma, hypertension, diabetes, hypercholesterolemia, and aging cause atherosclerosis and increased arterial stiffness and are associated with high vascular resistance and ventricular diastolic dysfunction (23). Over time, the long-term impact of cardiovascular risk factors on arterial stiffness causes LA dysfunction and induces atrial fibrosis, which also causes a poor LA expansion index (6, 8). Therefore, LA expansion index, a marker of atrial fibrosis, is a useful parameter for predicting recurrent ischemic stroke regardless of subtype. Progressive atrial fibrosis results in atrial stiffness and reduction of LA expansion index and is also a significant cause of AF $(5,6)$. In multiple Cox regression analyses of this study, both LA expansion index and $A F$, which indicate atrial fibrosis, are associated significantly with recurrent stroke. However, LA expansion index remains an independent predictor after excluding patients with AF (Table 4). 


\section{Study limitations}

This study had several limitations that merit discussion. Although this study had a five-year follow-up period, the event rate was relatively low. Therefore, it is unknown whether LA expansion index could be used to identify recurrent stroke subtypes. Furthermore, this study only investigated one cohort treated at a single center. Further large-scale multicenter studies are needed for getting adequate statistical power. A more detailed study is needed to provide precise data for arterial stiffness, atrial fibrosis, and the causal relationship between LA expansion index and recurrent stroke. Finally, other echocardiographic measures of LA function, including segmental atrial function, strain, strain rate, and atrial response to exercise were not examined. The predictive power among LA expansion index, strain rate/strain, and speckle tracking could not be compared.

\section{Conclusion}

The LA expansion index is useful for assessing recurrent stroke risk. As a predictor of recurrent stroke, LA expansion index is better than LA volume. A $10 \%$ increase in LA expansion index was associated with a $13 \%$ decrease in the five-year recurrent stroke rate.

Clinical Trial Registration: URL: http://www.clinicaltrials.gov. Unique identifier: NCT01171040.

Funding Support: This work was funded by the Ministry of Science and Technology (102-2628-B-650-002-MY3), Kaohsiung Veterans General Hospital (VGHKS11-CT3-02), and E-Da Hospital (EDAHP106026), Taiwan.

Conflict of interest: None declared.

Peer-review: Externally peer-reviewed.

Author contributions: Concept-S.H.H.; Design-S.H.H.; Supervision - S.H.H.; Fundings - S.H.H.; Materials - S.H.H.; Data collection \&/or processing - S.H.H.; Analysis \&/or interpretation - S.H.H.; Literature search - S.H.H.; Writing - S.H.H.; Critical review - S.H.H.

\section{References}

1. Di Tullio MR, Sacco RL, Sciacca RR, Homma S. Left atrial size and the risk of ischemic stroke in an ethnically mixed population. Stroke 1999; 30: 2019-24. [Crossref]

2. Benjamin EJ, D'Agostino RB, Belanger AJ, Wolf PA, Levy D. Left atrial size and the risk of stroke and death. The Framingham Heart Study. Circulation 1995; 92: 835-41. [Crossref]

3. Kizer JR, Bella JN, Palmieri V, Liu JE, Best LG, Lee ET, et al. Left atrial diameter as an independent predictor of first clinical cardiovascular events in middle-aged and elderly adults: the Strong Heart Study (SHS). Am Heart J 2006; 151: 412-8. [Crossref]

4. Bouzas-Mosquera A, Broullón FJ, Álvarez-García N, Méndez E, Peteiro J, Gándara-Sambade T, et al. Left atrial size and risk for all-cause mortality and ischemic stroke. CMAJ 2011; 183: E657-64. [Crossref]

5. King JB, Azadani PN, Suksaranjit P, Bress AP, Witt DM, Han FT, et al. Left atrial fibrosis and risk of cerebrovascular and cardiovascu- lar events in patients with atrial fibrillation. J Am Coll Cardiol 2017; 70: 1311-21. [Crossref]

6. Marrouche NF, Wilber D, Hindricks G, Jais P, Akoum N, Marchlinski $F$, et al. Association of atrial tissue fibrosis identified by delayed enhancement MRI and atrial fibrillation catheter ablation: the DECAAF study. JAMA 2014; 311: 498-506. [Crossref]

7. Jordan K, Yaghi S, Poppas A, Chang AD, Mac Grory B, Cutting S, et al. Left atrial volume Index is associated with cardioembolic stroke and atrial fibrillation detection after embolic stroke of undetermined source. Stroke 2019; 50: 1997-2001. [Crossref]

8. Yaghi S, Moon YP, Mora-McLaughlin C, Willey JZ, Cheung K, Di Tullio $M R$, et al. Left atrial enlargement and stroke recurrence: the Northern Manhattan Stroke Study. Stroke 2015; 46: 1488-93. [Crossref]

9. Tsang TS, Abhayaratna WP, Bames ME, Miyasaka Y, Gersh BJ, Bailey KR, et al. Prediction of cardiovascular outcomes with left atrial size: is volume superior to area or diameter? J Am Coll Cardiol 2006; 47: 1018-23. [Crossref]

10. Gupta S, Matulevicius SA, Ayers CR, Berry JD, Patel PC, Markham $D W$, et al. Left atrial structure and function and clinical outcome in the general population. Eur Heart J 2013; 34: 278-85. [Crossref]

11. Hsiao SH, Chu KA, Wu CJ, Chiou KR. Left Atrial Expansion Index Predicts Left Ventricular Filling Pressure and Adverse Events in Acute Heart Failure With Severe Left Ventricular Dysfunction. J Card Fail 2016; 22: 272-9. [Crossref]

12. Hsiao SH, Chiou KR. Left atrial expansion index predicts atrial fibrillation in dyspnea. Circ J 2013; 77: 2712-21. [Crossref]

13. Wang WH, Hsiao SH, Lin KL, Wu CJ, Kang PL, Chiou KR. Left atrial expansion index for predicting atrial fibrillation and in-hospital mortality after coronary artery bypass graft surgery. Ann Thorac Surg 2012; 93: 796-803. [Crossref]

14. Hart RG, Diener HC, Coutts SB, Easton JD, Granger CB, O'Donnell MJ, et al.; Cryptogenic Stroke/ESUS International Working Group. Cryptogenic Stroke/ESUS International Working Group. Embolic strokes of undetermined source: the case for a new clinical construct. Lancet Neurol 2014; 13: 429-38. [Crossref]

15. Dries DL, Exner DV, Domanski MJ, Greenberg B, Stevenson LW. The prognostic implications of renal insufficiency in asymptomatic and symptomatic patients with left ventricular systolic dysfunction. J Am Coll Cardiol 2000; 35: 681-9. [Crossref]

16. Rivas-Gotz C, Manolios M, Thohan V, Nagueh SF. Impact of left ventricular ejection fraction on estimation of left ventricular filling pressure using tissue Doppler and flow propagation velocity. Am J Cardiol 2003; 91: 780-4. [Crossref]

17. Ujino K, Barnes ME, Cha SS, Langins AP, Bailey KR, Seward JB, et al. Two-Dimensional echocardiographic methods for assessment of left atrial volume. Am J Cardiol 2006; 98: 1185-8. [Crossref]

18. Lang RM, Badano LP, Mor-Avi V, Afilalo J, Armstrong A, Ernande L, et al. Recommendations for cardiac chamber quantifications by echocardiography in adults: an update from the American Society of Echocardiography and the European Association of Cardiovascular Imaging. Eur Heart J Cardiovasc Imaging 2015; 16: 233-70. [Crossref]

19. Xue J, Lin Y, Huang W, Chen X, Li O, Cai Z, et al. Left atrial size and risk of recurrent ischemic stroke in a Chinese population. Brain Behav 2017; 7: e00702. [Crossref]

20. Ogata T, Matsuo R, Kiyuna F, Hata J, Ago T, Tsuboi Y, et al.; FSR Investigators. Left Atrial Size and Long-Term Risk of Recurrent Stroke After Acute Ischemic Stroke in Patients With Nonvalvular Atrial Fibrillation. J Am Heart Assoc 2017; 6: e006402. [Crossref] 
21. Boeckh-Behrens T, Kleine JF, Zimmer C, Neff F, Scheipl F, Pelisek J, et al. Thrombus Histology Suggests Cardioembolic Cause in Cryptogenic Stroke. Stroke 2016; 47: 1864-71. [Crossref]

22. Tandon K, Tirschwell D, Longstreth WT Jr, Smith B, Akoum N. Embolic stroke of undetermined source correlates to atrial fibro- sis without atrial fibrillation. Neurology 2019; 93: e381-7. [Crossref]

23. Mitchell GF, Hwang SJ, Vasan RS, Larson MG, Pencina MJ, Hamburg $N M$, et al. Arterial stiffness and cardiovascular events: the Framingham Heart Study. Circulation 2010; 121: 505-11. [Crossref] 